\title{
Fluctuation Electron Microscopy for the MASses
}

\author{
M.M.J. Treacy \\ Dept. of Physics and Astronomy, Arizona State University, Tempe, AZ 85287
}

The seeds of the fluctuation electron microscopy technique were sown in the early 1970s when speckle was observed in dark field TEM images of amorphous materials. The origin of the speckle in amorphous germanium was interpreted in terms of diffracting nanocrystallites [1], but this claim was disputed and largely discredited [2]. These early efforts operated on the premise that ordered regions of nanometer dimensions should diffract similarly to larger-scale polycrystalline regions, but with reduced intensity. Dark field images should show bright patches wherever a region is diffracting into the image-forming aperture. These straightforward ideas failed in the earlier studies of amorphous carbon, silicon and germanium for three reasons; (1) atomic ordering, if present, is on a very short scale $(<2 \mathrm{~nm})$, and is comparable in dimensions to the width of the point spread function of the microscope; (2) TEM images display a projection through the thinned sample, and these projections inevitably present a random average of any tiny ordered regions that may be present in typical sample thicknesses of $<30 \mathrm{~nm}$; (3) random alignments of widely separated atoms within projected columns can give the illusion of local Bragg scattering in TEM images when the scattering from such atoms is mutually coherent. The inability to distinguish speckle caused by real and accidental atomic alignments cast a long shadow over these earlier results.

Since the 1970s, the processing speed of computers has increased dramatically, and TEM images are now regularly acquired on specially adapted CCD cameras that offer high dynamic range and linearity. These developments set the stage for a quantitative approach to the problem of discerning structurally ordered patterns in TEM dark field images, Fluctuation Electron Microscopy (FEM), which has met with success in detecting the once-disputed medium-range order in amorphous silicon and germanium [3].

FEM is a refinement of the earlier dark-field speckle imaging methods. The key innovation was the recognition that the real structural alignments corresponding to medium-range order, and the random coherent scattering from accidental long-range alignments, can be discerned by statistical analysis of the speckle. The character of the speckle can be controlled by either adjusting the coherence volume by modifying the illumination conditions (variable coherence microscopy), or by adjusting the width of the point spread function by changing the objective aperture (variable resolution microscopy). In the FEM technique conducted in a conventional TEM, the coherence volume is usually controlled by using hollow-cone illumination. In the hollow-cone mode, the illuminating beam is tilted through an angle $\sim \lambda Q$ (where $\lambda$ is the electron wavelength, and $Q$ is a small horizontal momentum vector), and swept electronically so that the beam describes a cone with its apex at the specimen. This generates images from that apex region that are essentially the incoherent average of many tilted dark field images, with each tilt angle coming from the surface of the cone. This averaging has the effect of limiting the neighborhood around each atom wherein coherent scattering can occur, suppressing lateral long-range correlations. Smaller cone angles produce wider coherence volumes. The speckle characteristics can also be controlled by adjusting the size of the microscope point spread function. At low resolution, the point-spread function is adjusted by changing the diameter of the objective aperture $k$ (variable resolution microscopy). The smaller the objective aperture diameter, the wider the point spread function. The point-spread function has the effect of subdividing the sample into independent columns. Only atoms within each column can interfere with each other. By using two separate physical processes (illumination coherence, and image collection geometry), the thin film is effectively being subdivided into many 
small volumes containing typically $100-10,000$ atoms. The exact shape and size of the volume is controlled systematically by the microscope coherence and resolution. Each bright and dark speck on the image represents the independent scattering contribution from such (columnar) volumes. Ideally, sample thickness should be uniform so that all columns contain a comparable number of atoms.

Image data is gathered by a high quality CCD camera and downloaded to computer to facilitate analysis. Each image contains a wealth of information. However, for simplicity, the average intensity $\langle I(k, Q)\rangle$ (first moment) and the mean square intensity $\left\langle I^{2}(k, Q)\right\rangle$ (second moment) are computed for each image. The more speckly the image is, the higher the second moment. To eliminate dependencies on the material scattering factor and the total image exposure time, the normalized variance $V(k, Q)$ of the image intensity

$$
V(k, Q)=\frac{\left\langle I^{2}(k, Q)\right\rangle}{\langle I(k, Q)\rangle^{2}}-1
$$

is then computed [3]. Figure 1 shows a simple explanation of how dark field speckle can depend on the presence of medium range order.

In this talk, I will explain; (1) What is FEM. (2) Show how it works. (3) Show why it works. (4) Briefly describe recent advances in Fluctuation X-ray microscopy (FXM) and Fluctuation Optical Microscopy (FOM).

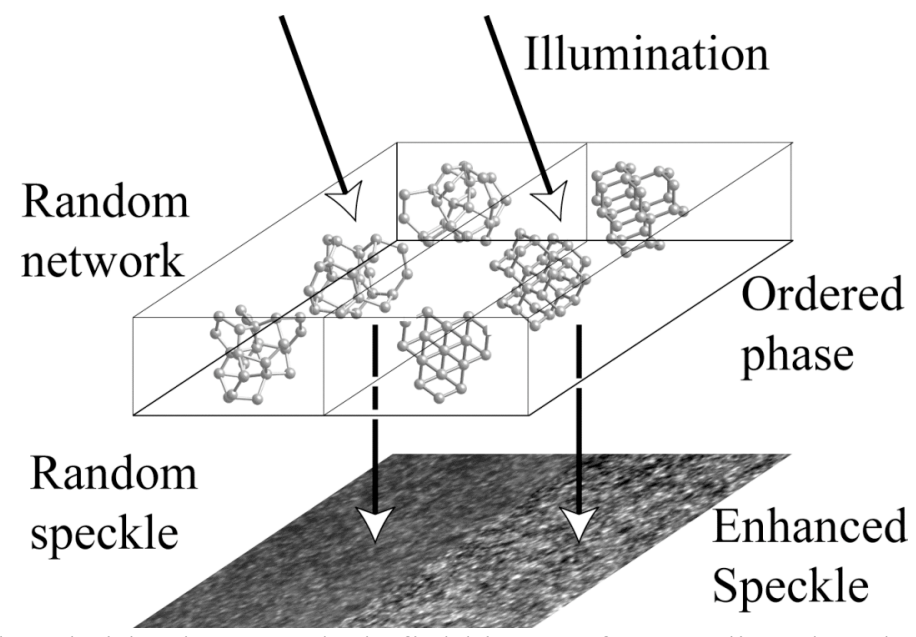

Figure 1: The left-hand side shows a dark-field image from a disordered specimen exhibiting speckle (image intensity fluctuations) due to random alignments of coherently scattering atoms. The right hand side shows a structure with paracrystalline medium range order with increased speckle because of random alignments of small locally ordered regions that are near a Bragg diffraction condition.

\section{References}

[1] Rudee, M.L. and A. Howie, Philos. Mag. 25, 1001 (1972).

[2] Chaudhari, P. and J.F. Graczyk. in 5th Int. Conf. on Amorphous and Liquid Semiconductors. Taylor and Francis, London, (1973).

[3] Treacy, M.M.J. and J.M. Gibson, Acta Cryst. A52, 212-220 (1996). 